\title{
Safety analysis of COVID-19 vaccines in liver transplant recipients: a two-center study
}

\author{
Rui Tang ${ }^{1}$, Chao $\mathrm{Li}^{1}$, Guangdong $\mathrm{Wu}^{1}$, Xuan Tong ${ }^{1}$, Lihan $\mathrm{Yu}^{1}$, Huayuan Hao ${ }^{1}$, Rui Liao ${ }^{2}$, Yucheng Hou ${ }^{1}$, \\ Jun Li ${ }^{1}$, Wenping Zhao', Tieyan Fan ${ }^{1}$, Leida Zhang ${ }^{2}$, Qian Lu ${ }^{1}$ \\ ${ }^{1}$ Hepatopancreatobiliary Center, Beijing Tsinghua Changgung Hospital, School of Clinical Medicine, Institute for Precision Medicine, Tsinghua \\ University, Beijing, China; ${ }^{2}$ Institute of Hepatobiliary Surgery, Southwest Hospital, Third Military Medical University (Amy Medical University), \\ Chongqing, China \\ Correspondence to: Qian Lu. Hepatopancreatobiliary Center, Beijing Tsinghua Changgung Hospital, School of Clinical Medicine, Institute for \\ Precision Medicine, Tsinghua University, No. 168 Litang Road, Beijing 102218, China. Email: luqian_lt@163.com.
}

Submitted Sep 18, 2021. Accepted for publication Dec 06, 2021.

doi: 10.21037/hbsn-21-392

View this article at: https://dx.doi.org/10.21037/hbsn-21-392

Up to know, product information for COVID-19 mRNA vaccines do not include their use in patients receiving immunosuppressive medication, since they were not included in trials (1). For the BNT162b2 mRNA vaccine, one case of otherwise totally asymptomatic elevations of alanine aminotransferase (ALT), aspartate aminotransferase (AST), alkaline phosphatase (ALP) and -glutamyl transpeptidase (GGT) serum concentrations in a liver transplant recipient has been reported after the first injection (2). In the present retrospective study, we present safety outcomes of COVID-19 vaccinations in 56 liver transplant patients from two Chinese centers vaccinated with the domestic PiCoVacc, BBIBP-CorV and ZF2001 vaccines. Thirty vaccinated physicians from the Hepatopancreatobiliary Center of Beijing Tsinghua Changgung Hospital served as control, from which 1 participant received an $\mathrm{Ad} 5-\mathrm{nCoV}$ vaccine. The study deadline was 2021-10-31. The observation period for adverse reactions, such as muscle pain and fatigue was 1 week, while for laboratory examinations such as liver function the follow-up period was 1 month.

PiCoVacc and BBIBP-CorV are both inactivated COVID-19 virus vaccines with a two-dose schedule and a spacing of 3 to 4 weeks, while ZF2001 is a recombinant tandem-repeat dimeric receptor-binding domain (RBD)based protein subunit vaccine with an application of three doses over a period of 2 months. $\mathrm{Ad} 5-\mathrm{nCoV}$ is a viral vector vaccine applied as a single injection. Table 1 shows the basic characteristics of the 86 participants. Adverse reactions in the liver transplant and control groups were fatigue (4, 7.1\% vs. 1, 3.3\%), local pain (9, 16.1\% vs. 6, 20.0\%), cough $(1,1.8 \%$ vs. 0$)$, rhinorrhea $(1,1.8 \%$ vs. 0$)$ and drowsiness ( 0 vs. $4,13.3 \%$ ), respectively. All adverse reactions were mild. Fifty-four participants had normal liver function tests within 1 month before and after vaccination. A 37-year-old patient, 70 months after transplantation due to a primary liver cancer who was receiving an antirejection regimen of sirolimus + mycophenolate mofetil (MMF), had liver enzyme values of ALT 72.0 U/L, AST 37.6 U/L, total bilirubin (TBIL) $13.7 \mu \mathrm{mol} / \mathrm{L}$, ALP $80.0 \mathrm{U} / \mathrm{L}$ and GGT 58.3 U/L before and ALT 68.3 U/L, AST 37.7 U/L, TBIL $11.0 \mu \mathrm{mol} / \mathrm{L}$, ALP 96.0 U/L and GGT 62.2 U/L after vaccination. The condition was considered to be related to the graft fatty liver and the liver function changes were not significant before and after vaccination. The second case was a 46-year-old patient with hepatic alveolar echinococcosis 30.7 months after transplantation and an anti-rejection regimen of tacrolimus + MMF. Liver enzyme values were ALT 22 U/L, AST 29 U/L, TBIL 12.4 mol/L, ALP 134 U/L, GGT 44 U/L before and ALT 19 U/L, AST 25 U/L, TBIL $11 \mu \mathrm{mol} / \mathrm{L}$, ALP $106 \mathrm{U} / \mathrm{L}$ and GGT $41 \mathrm{U} / \mathrm{L}$ after vaccination. The patient underwent a choledochojejunostomy, because of a narrow biliary anastomosis. Since there was no stenosis after the choledochojejunostomy, this abnormal ALP may be related to the state of bilioenteroanastomosis.

Two of the vaccines in the present study were inactivated viruses, which are only recommended for solid organ transplant recipients after careful risk benefit assessment, 
Table 1 Basic characteristics and vaccines of the participants

\begin{tabular}{|c|c|c|}
\hline Characteristic & Transplant group, $\mathrm{N}=56$ & Control group, $\mathrm{N}=30$ \\
\hline Male & 45 & 22 \\
\hline Female & 11 & 8 \\
\hline Age, years, median [range] & 49 [20-67] & $40[25-60]$ \\
\hline Polycystic liver & 2 & \\
\hline Liver cancer & 17 & \\
\hline Wilson's disease & 1 & \\
\hline Liver failure & 12 & \\
\hline Drug-induced liver injury & 3 & \\
\hline Anti-rejection regimen & & - \\
\hline MMF & 1 & \\
\hline Tacrolimus & 12 & \\
\hline Tacrolimus combined with MMF & 34 & \\
\hline Tacrolimus + sirolimus + MMF & 2 & \\
\hline Sirolimus & 1 & \\
\hline Sirolimus combined with MMF & 6 & \\
\hline \multicolumn{3}{|l|}{ Vaccines received } \\
\hline PiCoVacc vaccine & 39 & 27 \\
\hline BBIBP-CorV vaccine & 14 & 2 \\
\hline ZF2001 vaccine & 3 & 0 \\
\hline Ad5-nCoV vaccine & 0 & 1 \\
\hline
\end{tabular}

MMF, mycophenolate mofetil.

because of possible allosensitization, graft rejection and/ or infection $(3,4)$. However, a meta analysis of 90 studies reporting on standard (non-COVID-19) vaccination in transplant recipients showed a similar risk of transplant rejection compared with non vaccinated controls (3). Also in the present study, no graft rejection, infection or other serious vaccine related event occurred. A major concern is the lack of neutralizing antibody development after vaccination of transplant patients. As reported in the literature older age, use of high dose prednisone in the past 12 months, MMF and triple therapy, as well as poor renal function (low estimated glomerular filtration rate) were associated with low response to the vaccine (5). However, a third booster injection did improve the initially 
low titer and has been proposed to be incorporated into routine clinical practice (6), which is already the case for the ZF2001 vaccine. In addition, phase II trials of the BNT162b2 mRNA vaccine are under way in China, while booster injection of the inactivated vaccine is also being promoted. Liver transplant recipients will likely receive safe and effective protection from supplementary immunization and cocoon strategy.

\section{Acknowledgments}

Funding: This study was supported by the National Science \& Technology Major Project [grant No. 2017ZX100203205] and National Natural Science Foundation of China [grant No. 81930119]. The funders had no role in design of the study and collection, analysis, and interpretation of data and in writing the manuscript.

\section{Footnote}

Provenance and Peer Review: This article was a standard submission to the journal. The article has undergone external peer review.

Conflicts of Interest: All authors have completed the ICMJE uniform disclosure form (available at https://hbsn. amegroups.com/article/view/10.21037/hbsn-21-392/coif). The authors have no conflicts of interest to declare.

Ethical Statement: The authors are accountable for all aspects of the work in ensuring that questions related to the accuracy or integrity of any part of the work are appropriately investigated and resolved. The study was conducted in accordance with the Declaration of Helsinki (as revised in 2013). This study was approved by the Ethics Committee of Beijing Tsinghua Changgung Hospital (approval number: 21341-6-01). Donation of organs in this study reported was voluntary and with appropriate authorization or consent, and no executed prisoners served as organ donors.

Open Access Statement: This is an Open Access article distributed in accordance with the Creative Commons Attribution-NonCommercial-NoDerivs 4.0 International License (CC BY-NC-ND 4.0), which permits the noncommercial replication and distribution of the article with the strict proviso that no changes or edits are made and the original work is properly cited (including links to both the formal publication through the relevant DOI and the license). See: https://creativecommons.org/licenses/by-nc-nd/4.0/.

\section{References}

1. Marjot T, Webb GJ, Barritt AS, et al. SARS-CoV-2 vaccination in patients with liver disease: responding to the next big question. Lancet Gastroenterol Hepatol 2021;6:156-8.

2. Dumortier J. Liver injury after mRNA-based SARS$\mathrm{CoV}-2$ vaccination in a liver transplant recipient. Clin Res Hepatol Gastroenterol 2022;46:101743.

3. Mulley WR, Dendle C, Ling JEH, et al. Does vaccination in solid-organ transplant recipients result in adverse immunologic sequelae? A systematic review and metaanalysis. J Heart Lung Transplant 2018;37:844-52.

4. Croce E, Hatz C, Jonker EF, et al. Safety of live vaccinations on immunosuppressive therapy in patients with immune-mediated inflammatory diseases, solid organ transplantation or after bone-marrow transplantation A systematic review of randomized trials, observational studies and case reports. Vaccine 2017;35:1216-26.

5. Rabinowich L, Grupper A, Baruch R, et al. Low immunogenicity to SARS-CoV-2 vaccination among liver transplant recipients. J Hepatol 2021;75:435-8.

6. Werbel WA, Boyarsky BJ, Ou MT, et al. Safety and Immunogenicity of a Third Dose of SARS-CoV-2 Vaccine in Solid Organ Transplant Recipients: A Case Series. Ann Intern Med 2021;174:1330-2.
Cite this article as: Tang R, Li C, Wu G, Tong X, Yu L, Hao H, Liao R, Hou Y, Li J, Zhao W, Fan T, Zhang L, Lu Q. Safety analysis of COVID-19 vaccines in liver transplant recipients: a two-center study. HepatoBiliary Surg Nutr 2022;11(1):166-168. doi: 10.21037/hbsn-21-392 